\title{
Interplay between genotoxic factors formaldehyde and UV-B exacerbates genome instability in Arabidopsis
}

\author{
Yu Wang ${ }^{1}$, Jinzheng WANG ${ }^{2}$, Qiang $\mathrm{Lv}^{1}$, and Yi-Kun $\mathrm{He}^{1}$ \\ ${ }^{1}$ Capital Normal University \\ ${ }^{2}$ University of California Riverside
}

May 20, 2021

\begin{abstract}
Maintenance of genome stability is quintessential feature for all living organisms. The simplest aldehyde formaldehyde and UV-B radiation, two environmental toxic factors, cause DNA damage, affect genome stability, subsequently growth and development across kingdoms. However, the interrelationship of genotoxicity caused by formaldehyde and UV-B remains fragmented in plants. Here, we show that mutants lacking one aldehyde detoxifying enzyme, alcohol dehydrogenase 2 (ADH2, also named GSNOR/FALDH), are hypersensitive to low dosage UV-B radiation or UV-B radiation-mimetic chemical in seedling and root growth. The defects are not caused by the alteration of UV-B sensing, secondary metabolites flavonoid accumulation, or ROS accumulation, rather are UV-B-induced genotoxicity. Increased DNA damage response genes and comet assay tail, cell cycle arrest upon exposure to UV-B provide direct evidence for DNA damage in gsnor mutant. Pharmacological analyses show that the susceptibility to genotoxic stresses is caused by the increased DNA crosslink which results from the enhanced endogenous formaldehyde in gsnor while UV-B promotes the production of formaldehyde. This implies formaldehyde clearance through GSNOR plays a critical role in response to environmental genotoxic stress and interplay between formaldehyde and UV-B exacerbates genome instability.
\end{abstract}

\section{Hosted file}

GSNOR_final_PCE.pdf available at https://authorea.com/users/414813/articles/522731-interplaybetween-genotoxic-factors-formaldehyde-and-uv-b-exacerbates-genome-instability-inarabidopsis 